\title{
Forced oscillations in the clinical setting in young children with neonatal lung disease
}

\author{
K. Udomittipong*,\#, P.D. Sly*,", H.J. Patterson*, C.L. GangelI ${ }^{+}$, \\ S.M. Stick ${ }^{\uparrow,+}$ and G.L. Hall ${ }^{\uparrow,+}$
}

ABSTRACT: The extent of respiratory dysfunction is not well characterised in children with neonatal chronic lung disease (nCLD) too young to perform spirometry. Forced oscillations are easily performed by healthy young children; however, they may be more difficult for those with $\mathrm{nCLD}$. The present study aimed to describe the feasibility of using the forced oscillation technique in children with nCLD in a routine clinical setting and to investigate the influence of neonatal factors on subsequent lung function.

Respiratory function tests were attempted in 64 patients with nCLD aged 3.2-6.6 yrs. Respiratory resistance and reactance at 6,8 and $10 \mathrm{~Hz}$ were expressed as z-scores derived from a healthy reference population. The within-test variation and between-test repeatability were also assessed.

Technically, satisfactory data were obtained from $77 \%$ of children. On grouped data, z-scores for all oscillatory indices were different from zero and related to hospital oxygen administration in the neonatal period.

In conclusion, the forced oscillation technique was feasible in preschool children with neonatal chronic lung disease in the clinical outpatient setting. These children had lung function significantly worse than that predicted from healthy children. Respiratory function assessed using forced oscillations appeared to reflect the severity of lung disease during the neonatal period.

KEYWORDS: Forced oscillations, lung function, neonatal chronic lung disease, paediatrics, preschool children

$\mathbf{N}$ eonatal chronic lung disease (nCLD) is the most common chronic respiratory disease in children and is primarily related to premature birth and injury from the required oxygen therapy and mechanical ventilation [1]. Advances in neonatal care, including antenatal corticosteroids and post-natal surfactant therapy, have resulted in a gradual but significant change in the pathogenesis, epidemiology and clinical syndrome of nCLD [2]. Previously, nCLD was characterised by severe airway epithelial lesions, airway smooth muscle hyperplasia, extensive fibroproliferation and a reduced number of alveoli [2]. During the post 1990s, infants with nCLD were more premature, had less prominent airway pathology and instead exhibited increased abnormalities of the lung periphery, including failed alveolarisation with relatively fewer, larger, simplified alveoli [3]. The resulting respiratory dysfunction may persist through to adolescence, and in severely affected patients, chronic obstructive lung disease may develop in adulthood [4]. Many studies of respiratory function in infants [5-8], school children [9-12] and adolescents with a history of nCLD [13, 14] have demonstrated low lung function. However, relatively few studies have documented the severity of respiratory dysfunction among those children with a history of nCLD born in the post 1990s, and the majority of respiratory function techniques used were insensitive to alterations of intraparenchymal airway and lung tissue mechanics as seen in the "new" nCLD group [3].

The forced oscillation technique (FOT) allows the measurement of the respiratory system impedance (Zrs) and its components respiratory resistance $(R \mathrm{rs})$ and respiratory reactance (Xrs). $R$ rs includes the airway, lung tissue and chest wall resistance, while Xrs represents the balance of respiratory elastance and inertance [15]. FOT has been shown to be feasible in children as young as $3 \mathrm{yrs}$ old and can be performed in a routine clinical setting [16, 17]. FOT has previously been used in two studies of children with nCLD [18, 19]. MALMBERG et al. [18] reported a comprehensive assessment of respiratory function
AFFILIATIONS

*Centre for Child Health Research,

${ }^{+}$School of Paediatrics and Child Health, University of Western

Australia, and

"Respiratory Medicine, Princess Margaret Hospital, Perth, WA, Australia.

\#Dept of Paediatrics, Faculty of Medicine, Siriraj Hospital, Mahidol University, Bangkok, Thailand.

CORRESPONDENCE

G.L. Hall

Respiratory Medicine

Princess Margaret Hospital for

Children

GP0 Box D184

Perth

Australia 6840

Fax: 61893408181

E-mail: graham.hall@

health.wa.gov.au

Received:

May 142007

Accepted after revision:

January 222008

SUPPORT STATEMENT

$\mathrm{K}$. Udomittipong was funded by the Siriraj Hospital, Bangkok, Thailand. P.D. Sly and S.M. Stick were funded by the National Health and Medical Research Council, Canberra, ACT, Australia.

STATEMENT OF INTEREST None declared.

European Respiratory Journal Print ISSN 0903-1936 Online ISSN 1399-3003 
using both conventional methods (spirometry, static lung volumes and gas transfer) and FOT in children at a mean age of 8 yrs. VRIJLANDT et al. [19] used FOT and the interrupter technique in a group of pre-term children with and without nCLD and age-matched term controls aged 3-5 yrs. Both studies reported worsening $R$ rs and $X$ rs values in children both with and without nCLD compared with healthy controls; however, neither study examined the potential effect of neonatal factors on subsequent preschool respiratory function.

The FOT has not been applied in a population of young children with nCLD in a clinical outpatient setting. Young children with nCLD are more likely to have some degree of intellectual and motor impairment than their healthy counterparts and, therefore, less able to produce technically acceptable FOT measurements. The test results may also be more variable, both within a testing session and between tests repeated at short intervals, for example when measuring bronchodilator (BD) responses.

In a prospectively recruited group of young children with nCLD presenting at a tertiary paediatric respiratory outpatient clinic the present authors aimed to investigate the feasibility and short-term repeatability of measuring lung function using FOT. Furthermore, the influence of perinatal factors predicting lung function during preschool age in this group was examined.

\section{METHODS}

\section{Subjects}

Children with a neonatal history of nCLD, born between 1998 and 2002, aged 3-7 yrs and attending the respiratory outpatient clinics at Princess Margaret Hospital for Children (Perth, WA, Australia), between May 2004 and July 2005 were studied. All children were clinically stable and without respiratory tract infections in the previous 2 weeks. nCLD was defined as the use of supplemental oxygen for $>28$ days at 36 weeks post-menstrual age for infants with gestational age (GA) at birth of $<32$ weeks, and the use of supplemental oxygen at 28 days of life for individuals with GA at birth $\geqslant 32$ weeks [20].

Neonatal information was obtained from the children's medical records and included GA, birth weight, Apgar scores at $5 \mathrm{~min}$, surfactant administration, the number of infections, the presence of pneumothorax (PTX), diaphragmatic hernia (DPH), hyaline membrane disease (HMD) and pulmonary interstitial emphysema (PIE), the duration of conventional mechanical ventilation (CMV) and continuous positive airway pressure (CPAP), and days of supplemental oxygen therapy in hospital and at home. Body weight and height at the time of lung function were recorded, both in absolute and z-score terms [21]. Recent inhaled corticosteroid (ICS) and BD use, as well as respiratory symptoms, were also obtained from a retrospective review of the child's paediatric medical records.

\section{Protocol}

Measurements of forced oscillatory mechanics were performed as previously described [17] and in agreement with recent international FOT recommendations [15, 22]. Briefly, during respiratory function testing, children were in a sitting position with the head in a neutral position. Zrs spectra were measured using a commercially available system (i2m; Chess Medical Technology, Ghent, Belgium) and were collected using a pseudo-random forcing signal, containing integer multiple components between $2-48 \mathrm{~Hz}$. The forcing signal was applied to the respiratory system via a mouthpiece (RJVB2, SureGard bacterial filter; Bird Healthcare, Port Melbourne, VIC, Australia) during tidal breathing. A nose-clip was applied and the child's cheeks and lower jaw were supported by the hands of the investigator. For each child, three to five acceptable measurements were obtained, and the mean and SD for Rrs and Xrs at 6, 8 and $10 \mathrm{~Hz}$ (denoted as Rrsf or Xrsf, where $\mathrm{f}$ is the frequency) were reported. Measurements were considered technically acceptable if the child adopted a regular breathing pattern without artefacts introduced by speaking, coughing, swallowing, glottic closure or leak around the mouthpiece. In addition, if the coherence of three or more individual frequency components was $<0.95$, the measurement was rejected.

To characterise the short-term repeatability of the FOT in children with nCLD, two measurement sets were obtained $\sim 15$ min apart. BD responsiveness data from this group of children were also assessed and the results published elsewhere [23].

\section{Statistical analysis}

The distributions of continuous data were tested using a Shapiro-Wilk test and are described as mean \pm SD or median (10th-90th percentiles) for normal and non-normal distributions, respectively.

Baseline forced oscillatory variables were expressed as z-scores derived from a local population of 158 healthy preschool children, aged 2-7 yrs, height range $92-127 \mathrm{~cm}$, in whom Zrs was measured using an identical FOT protocol. These healthy children had no history of parentally reported wheeze or asthma at any time of their life and no acute respiratory infections within the past 3 weeks, and are described in detail elsewhere [17].

The feasibility of FOT measurements in young children with a history of nCLD was assessed from the children's first attempt at FOT testing. A test was considered successful if a minimum of three acceptable measurements could be obtained. The within-test variation of oscillatory variables was calculated using the coefficient of variation of baseline lung function, computed from SD divided by the mean of the acceptable measurements. The between-test repeatability in both absolute and z-score terms was evaluated using Bland and Altman analysis [24]. The coefficient of repeatability was determined as twice the SD of the differences between measurements.

Associations between neonatal and paediatric factors and the derived z-score for each respiratory function variable were evaluated by Spearman correlations for continuous data. The influence of neonatal (PTX, PIE, DPH and HMD) and respiratory (ICS and $\mathrm{BD}$ use and recent symptoms) factors on respiratory function variables were analysed with two-tailed, unpaired, independent $t$-tests considering these data as binary variables (present/absent). Those factors found to influence respiratory function at a statistical level of $p<0.1$ were included in a stepwise multiple linear regression model in which $p$-values $\leqslant 0.05$ were considered statistically significant. 


\begin{tabular}{lc} 
TABLE $\mathbf{1}$ Neonatal characteristics of study population \\
Subjects (M/F) $\mathbf{n}$ & $49(23 / 26)$ \\
Gestational age weeks & $26(24-30)$ \\
Birth weight $\mathbf{g}$ & $860(630-1175)$ \\
Apgar at $\mathbf{5}$ min & $8(5-9)$ \\
Surfactant administration & $38(78)$ \\
Neonatal conditions ${ }^{\#}$ & \\
PTX & $3(6)$ \\
DPH & $11(23)$ \\
PIE & $4(9)$ \\
HMD & $41(87)$ \\
Neonatal infection & $29(64)$ \\
Number of infections & $1(0-4)$ \\
Duration of treatment days & \\
CMV & $11(1-51)$ \\
CPAP & $9(2-30)$ \\
CMV and CPAP & $30(5-64)$ \\
Oxygen & \\
In hospital & $87(53-130)$ \\
At home & $65(25-158)$ \\
Total & $157(91-302)$ \\
\hline
\end{tabular}

Data are presented as median (10th-90th percentiles) or $n(\%)$, unless otherwise stated. M: male; F: female; PTX: pneumothorax; DPH: diaphragmatic hernia; PIE: pulmonary interstitial emphysema; HMD: hyaline membrane disease; CMV: conventional mechanical ventilation; CPAP: continuous positive airway pressure. ${ }^{\#}: n=47 ;{ }^{\natural}: n=45$.

\section{RESULTS}

The neonatal characteristics of the study population are shown in table 1, while demographic data of the group at the time of FOT testing are shown in table 2. At the time of testing, no children had recent respiratory tract infections or presence of respiratory signs on auscultation, as noted by the attending physician.

\section{Feasibility}

Zrs measurements were attempted in 64 children with a history of nCLD, with technically acceptable baseline measurements

\begin{tabular}{lc} 
TABLE 2 & $\begin{array}{l}\text { Demographic data of the children at the time of } \\
\text { testing }\end{array}$ \\
Subjects (M/F) $\mathbf{n}$ & $49(23 / 26)$ \\
Age yrs & $5.17(3.6-5.9)$ \\
Height $\mathbf{c m}$ & $106(96-113)$ \\
Height z-score & $-0.46(-1.79-0.41)$ \\
Weight kg & $16.4(13.9-19.9)$ \\
Weight z-score & $-0.42(-2.01-0.65)$ \\
Recent treatment & \\
ICS & $8(18)$ \\
BD & $13(29)$ \\
Recent respiratory symptoms & $28(62)$ \\
\hline
\end{tabular}

Data are presented as median (10th-90th percentiles) or $n$ (\%), unless otherwise stated. M: male; F: female; ICS: inhaled corticosteroid; BD: bronchodilator. ${ }^{*}$ : derived from British growth percentiles [21]; $" \mathrm{n}=45$. obtained from 49 (77\%) children. The proportion of children successfully performing FOT increased with age; being 38\% (six out of 16) in 3-yr-old children, 78\% (18 out of 23 ) in 4-yrolds and $100 \%$ (25 out of 25 ) in children aged $\geqslant 5$ yrs.

The median (10th-90th percentiles) post-natal age of children who were able to successfully complete oscillatory measurements was 5.17 (3.6-5.9) yrs, with the youngest child being 3.2 yrs old.

\section{Baseline and repeated lung function}

The group mean data of all respiratory function variables were significantly worse than the healthy reference group $(\mathrm{p}<0.001)$, with increased Rrs and decreased Xrs. The within-test variations of $\operatorname{Rrs}$ and $X \mathrm{rs}$ ranged between $5-7 \%$ and $12-14 \%$, respectively. Absolute and predicted respiratory function data and within-test variation results are shown in table 3 . Figure 1 shows the mean Rrs6 and Xrs6 for individual children.

The short-term repeatability protocol was attempted by 37 children, with paired data sets obtained from 19 (51\%) children. The between-test repeatability revealed no systematic bias, with mean difference between the two sets of measurements close to zero for all respiratory function indices (table 3). Individual differences were equally scattered around the mean difference, and not related to the magnitude of the measurement, subject height or age. The Bland and Altman plots for Rrs6 and Xrs6 are shown in figure 2.

\section{Associations between clinical factors and lung function}

Neonatal factors found to influence FOT variables in a univariate analysis were the presence of HMD (Rrs and $X_{\mathrm{rs}}$ at all frequencies; $\mathrm{p}<0.01)$; duration of CMV (Xrs6; $\mathrm{p}<0.1)$ or ventilation support (CMV and CPAP; Xrs6 and Xrs8; $\mathrm{p}<0.1)$; duration of oxygen supplementation in hospital (Rrs and $X$ rs at all frequencies; $\mathrm{p}<0.01)$; and total supplemental oxygen duration (Xrs6, Xrs8 and Xrs10; $\mathrm{p}<0.1$ ).

Recent inhaled therapy (ICS or BD) use or parentally reported respiratory symptoms did not significantly alter FOT z-scores $(\mathrm{p}>0.3)$. There were no relationships between age, height, weight or sex and respiratory function. Using multiple linear regression modelling, the only clinical factor found to contribute significantly to subsequent respiratory function was duration of oxygen therapy in hospital, with increasing duration significantly associated with worsening Rrs and Xrs (table 4). The relationships between duration of oxygen therapy in hospital and Rrs6 and Xrs6 are shown in figure 3.

\section{DISCUSSION}

The present study is the first to report the use of the FOT in a group of young children with a history of nCLD and prematurity, attending paediatric respiratory outpatient clinics. In addition, associations are reported between neonatal oxygen therapy duration and subsequent lung function. It has been demonstrated that the use of FOT to obtain objective measurements of respiratory function is feasible in this group in a clinical outpatient setting with acceptable within-test variations, similar to those reported in other patient groups of the same age. Repeatability of the oscillatory mechanics over a 15 -min period verified that there was no systematic bias of measurements, demonstrating that the FOT is a stable 

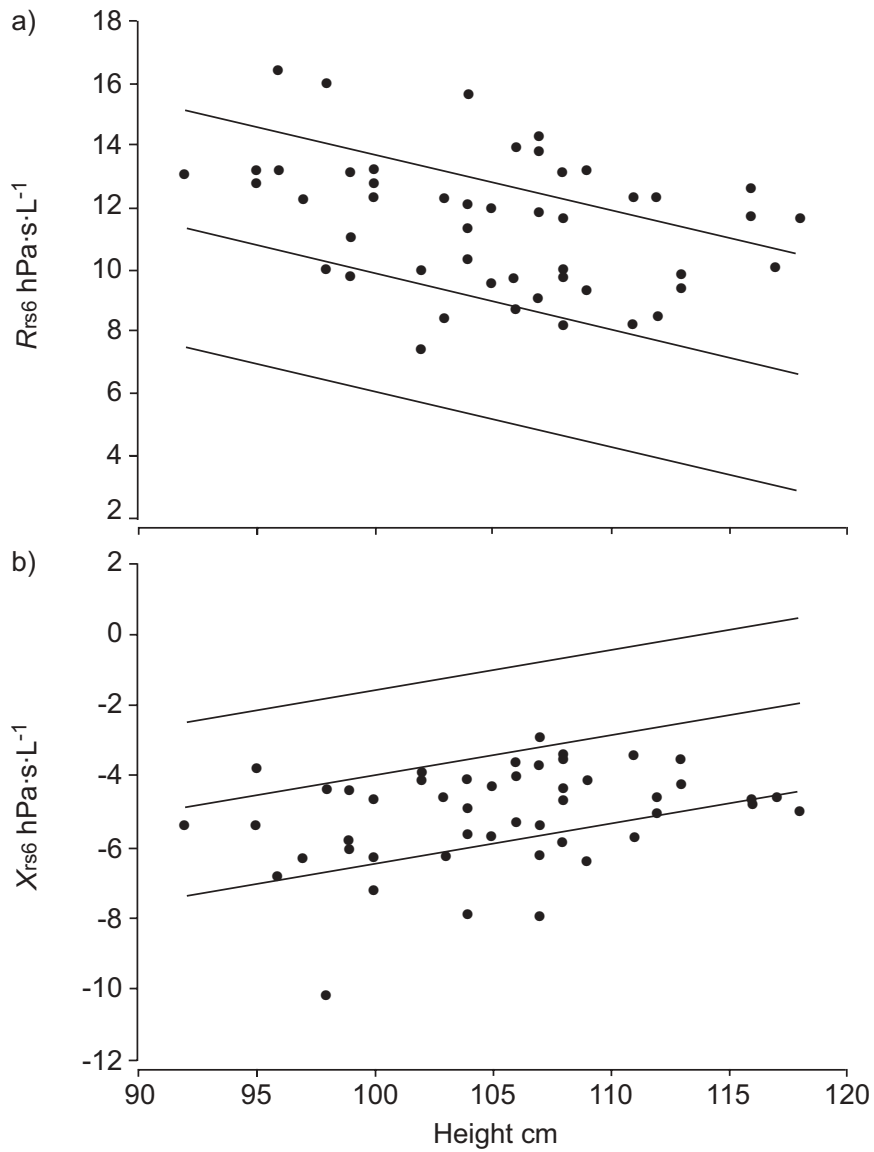

FIGURE 1. a) Respiratory resistance at $6 \mathrm{~Hz}$ (Rrs6) and b) respiratory reactance at $6 \mathrm{~Hz}$ (Xrs6) in relation to height in 49 young children with a history of neonatal chronic lung disease. Data are plotted as individual mean measurements.

—: predicted, upper and lower limits of normal ( \pm 2 z-scores) derived from a healthy reference population

measurement method in these young children with nCLD within the outpatient setting. Both airway and respiratory tissue mechanics of this population of young children with nCLD were significantly worse compared with healthy children, with increased Rrs and decreased Xrs being demonstrated. Respiratory function at preschool age was found to be related to the duration of oxygen administration in the neonatal period, suggesting that lung function measured with FOT is sensitive to the severity of neonatal lung disease.

\section{Feasibility}

The present study demonstrated that the FOT is feasible for use in young children with a history of nCLD in the clinical setting and that older children were more likely to be able to master the technique. Previous studies in healthy preschool children showed a comparable success rate in children aged $\geqslant 4$ yrs, but a greater feasibility in children aged 3 yrs (65 versus $38 \%$ ) [25]. The lower success rate in young children with nCLD may be due to intellectual, neuro-developmental delay and/or motor impairments, which are recognised sequelae of pre-term birth. These delays may result in a shift to lower agerelated success rates when compared with healthy children. In the present study, developmental delay was not quantified; therefore, the authors cannot comment directly on this effect. It is likely that with subsequent clinical visits and increased familiarisation with the laboratory and testing requirements, feasibility of successful measurements would increase. Indeed, in a population of young children with cystic fibrosis (CF) in the outpatient setting, it was reported that successful FOT measurements could be obtained, on average, after two or three visits [26]. DUCHARME and DAvis [27] reported successful measurements of respiratory function using the FOT in $65 \%$ of preschool children with acute asthma presenting to the emergency department, and also showed progressively greater success rates with increasing age: $19 \%$ for age $3 \mathrm{yrs} ; 40 \%$ for $4 \mathrm{yrs}$; and $83 \%$ for $5 \mathrm{yrs}$. The lower success rates in obtaining acceptable FOT measurements reported by DUCHARME and DAVIS [27] are understandable, as the children performed lung function during an acute respiratory illness.

\section{Repeatability}

Based on the present study, FOT in young children with nCLD yields clinically acceptable within-test variation and betweentest repeatability. The within-test variation of Rrs6 and Rrs8 ranged between $5-7 \%$ and is comparable with the previous reports of $5-10 \%[17,18,25,28]$. Reactance exhibited a withintest variation of $14 \%$ for both $\mathrm{Xrs} 6$ and $\mathrm{Xrs} 8$, similar to values of $17-20 \%$ reported in healthy children [17] and $16-17 \%$ reported by BISGAARD and KLUG [29, 30].

TABLE 3 Mean baseline respiratory function and between test repeatability

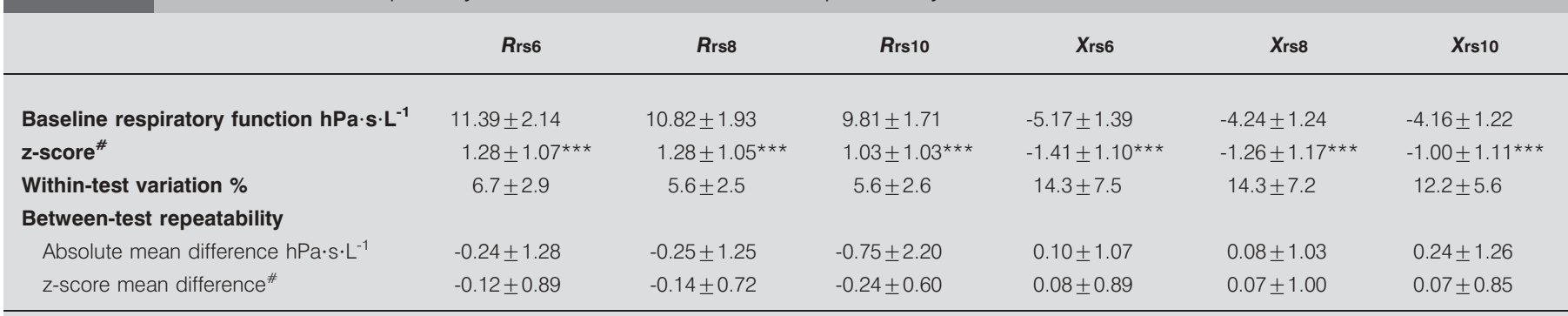

Data are expressed as mean \pm SD. Rrs6, Rrs8, Rrs10: respiratory system resistance at 6, 8 and $10 \mathrm{~Hz}$, respectively; $\mathrm{Xrs}_{\mathrm{rs}}, \mathrm{Xrs}_{\mathrm{r} 8}, \mathrm{X}_{\mathrm{rs} 10}$ : respiratory system reactance at 6, 8 and $10 \mathrm{~Hz}$, respectively. ${ }^{*}$ : expressed in standardised SD units. ${ }^{* * *}: \mathrm{p}<0.001$ for comparison with reference group [17]. 

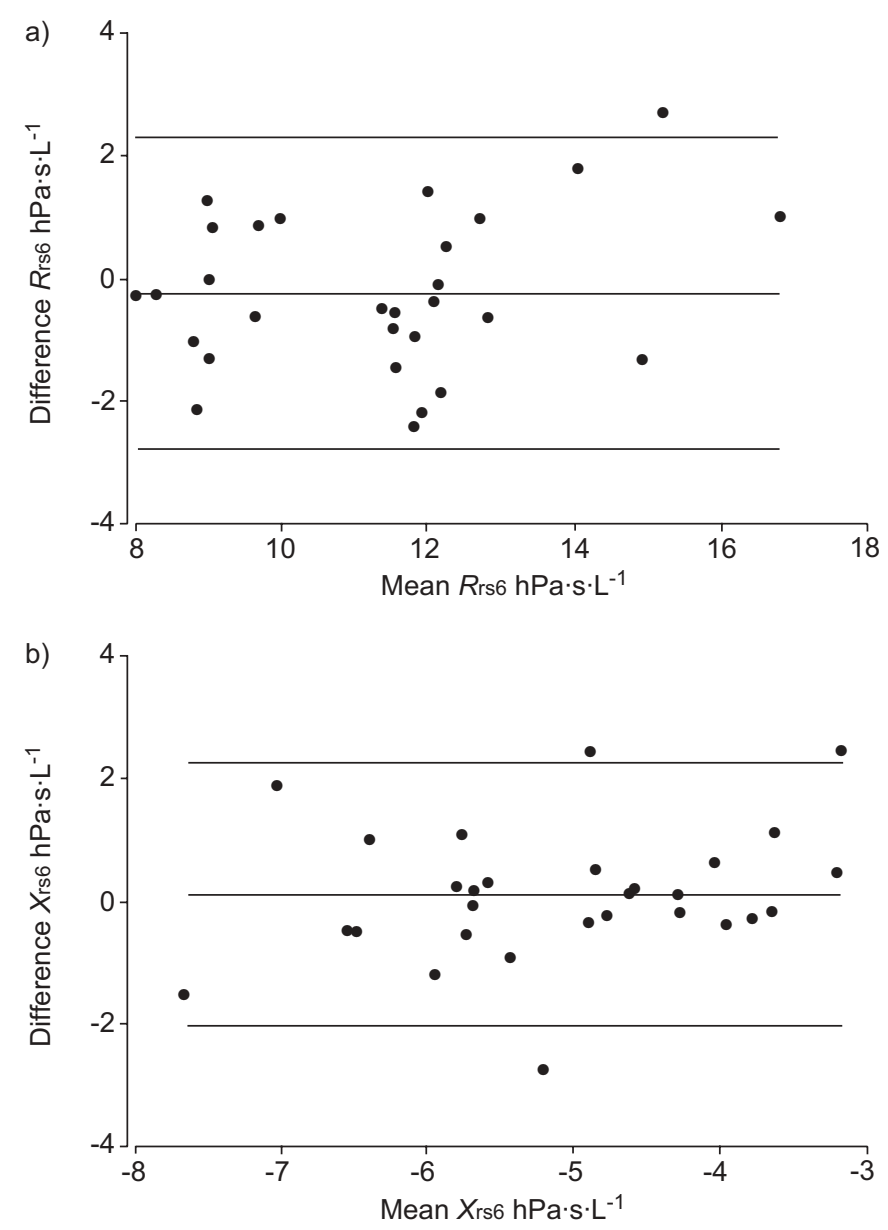

FIGURE 2. Bland and Altman plots for a) respiratory resistance at $6 \mathrm{~Hz}$ (Rrs6) and b) respiratory reactance at $6 \mathrm{~Hz}$ (Xrs6), comparing measurement sets $\sim 15 \mathrm{~min}$ apart for repeatability. Data are plotted as the difference between measurement sets versus the mean of the two measurement sets. - : mean of the difference and the upper and lower limits of agreement.

Bland and Altman analysis of between-test repeatability showed a mean difference between the two sets of measurements close to zero, indicating no systematic bias was introduced; this is in agreement with previous studies [17, $18,25,26]$. The coefficients of repeatability in the current study were 2.6 and $2.1 \mathrm{hPa} \cdot \mathrm{s} \cdot \mathrm{L}^{-1}$ for $R \mathrm{rs} 6$ and $\mathrm{Xrs6}$, respectively, similar to coefficients of repeatability in healthy young children (1.8-2.0 and $1.2-1.7 \mathrm{hPa} \cdot \mathrm{s} \cdot \mathrm{L}^{-1}$ for Rrs and Xrs, respectively) and children with CF (2.1-2.5 and 1.3$1.5 \mathrm{hPa} \cdot \mathrm{s} \cdot \mathrm{L}^{-1}$ for $R \mathrm{rs}$ and $\mathrm{Xrs}$, respectively) $[17,26]$. MALMBERG et al. [18] reported coefficients of repeatability of 0.8 and $0.9 \mathrm{hPa} \cdot \mathrm{s} \cdot \mathrm{L}^{-1}$ for $\operatorname{Rrs} 5$ and $\mathrm{Xrs5}$, respectively, in 19 children following placebo inhalation, while KLUG and BISGAARD [25] reported coefficients of repeatability in 120 children aged 27 yrs for Rrs5 and Xrs5 of 2.6 and $2.0 \mathrm{hPa} \cdot \mathrm{s} \cdot \mathrm{L}^{-1}$, respectively.

\section{Resistance and reactance}

In the present study, preschool-aged nCLD children had significantly worse respiratory function than those reported in a local, contemporary, age- and height-matched healthy population [17], as evidenced by the increased Rrs and

\begin{tabular}{|c|c|c|c|}
\hline \multirow[t]{2}{*}{ TABLE 4} & \multicolumn{3}{|c|}{$\begin{array}{l}\text { Stepwise multiple linear regression analysis } \\
\text { demonstrated association between the duration } \\
\text { of hospital oxygen administration and respiratory } \\
\text { function later in life }\end{array}$} \\
\hline & $\mathrm{R}^{2}$ & B & $p$-value \\
\hline Rrs6 & 0.224 & 0.473 & 0.006 \\
\hline Rrs8 & 0.186 & 0.431 & 0.014 \\
\hline Rrs10 & 0.182 & 0.427 & 0.015 \\
\hline Xrs6 & 0.150 & -0.388 & 0.028 \\
\hline Xrs8 & 0.194 & -0.440 & 0.012 \\
\hline Xrs10 & 0.142 & -0.377 & 0.033 \\
\hline
\end{tabular}

$R^{2}$ : R-square coefficient of determination; B: standardised regression coefficient; Rrs6, Rrs8, Rrs10: respiratory system resistance at 6,8 and $10 \mathrm{~Hz}$, respectively; $X_{r s 6}, X_{r s} 8, X_{r s 10:}$ respiratory system reactance at 6,8 and $10 \mathrm{~Hz}$, respectively.

decreased Xrs group mean z-scores, respectively. The present authors recognise that children born prematurely are likely to be shorter for their post-natal age; indeed, the median height zscore for age of this group was -0.46 . However, this is not likely to explain the abnormalities in lung function reported in the present study.

The altered lung function reported in the current study is similar to that of VRIJLANDT et al. [19] in young children and of MALmberg et al. [11] in older children. VRIJLANDT et al. [19] reported FOT mechanics in young children both with and without a history of nCLD, demonstrating increased Rrs and decreased Xrs in both groups when compared with a healthy control group. Interestingly, the only significant difference between pre-term children with and without nCLD was in measures of reactance, suggesting an increased involvement of peripheral lung mechanics in pre-term children with nCLD. MALmberg et al. [11] reported poor Rrs and Xrs values in children born prematurely with a history of nCLD at an average age of $8 \mathrm{yrs}$. These children also demonstrated reduced gas exchange, suggesting impairments of alveolar development as previously proposed [3]. However, neither of these studies tested the feasibility of using FOT within the clinical outpatient setting or examined the potential effects of neonatal factors on subsequent preschool respiratory function.

In the present study, there was a tendency for Rrs and Xrs to deviate further from normality at lower frequencies; i.e. mean $\mathrm{z}$-scores at $6 \mathrm{~Hz}$ were shifted further from normal than z-scores at $10 \mathrm{~Hz}$. Reactance reflects the combination of elastance and inertance. The influence of elastance decreases as the frequency of the forcing function increases; however, the frequency range used in the current study $(6-10 \mathrm{~Hz})$ is too small to make meaningful comments about reflections of physiological properties revealed by the different frequencies.

\section{Correlation between neonatal factors and subsequent lung function}

The period from 24 weeks gestation until birth is clinically significant, encompassing the canalicular, saccular and alveolar 

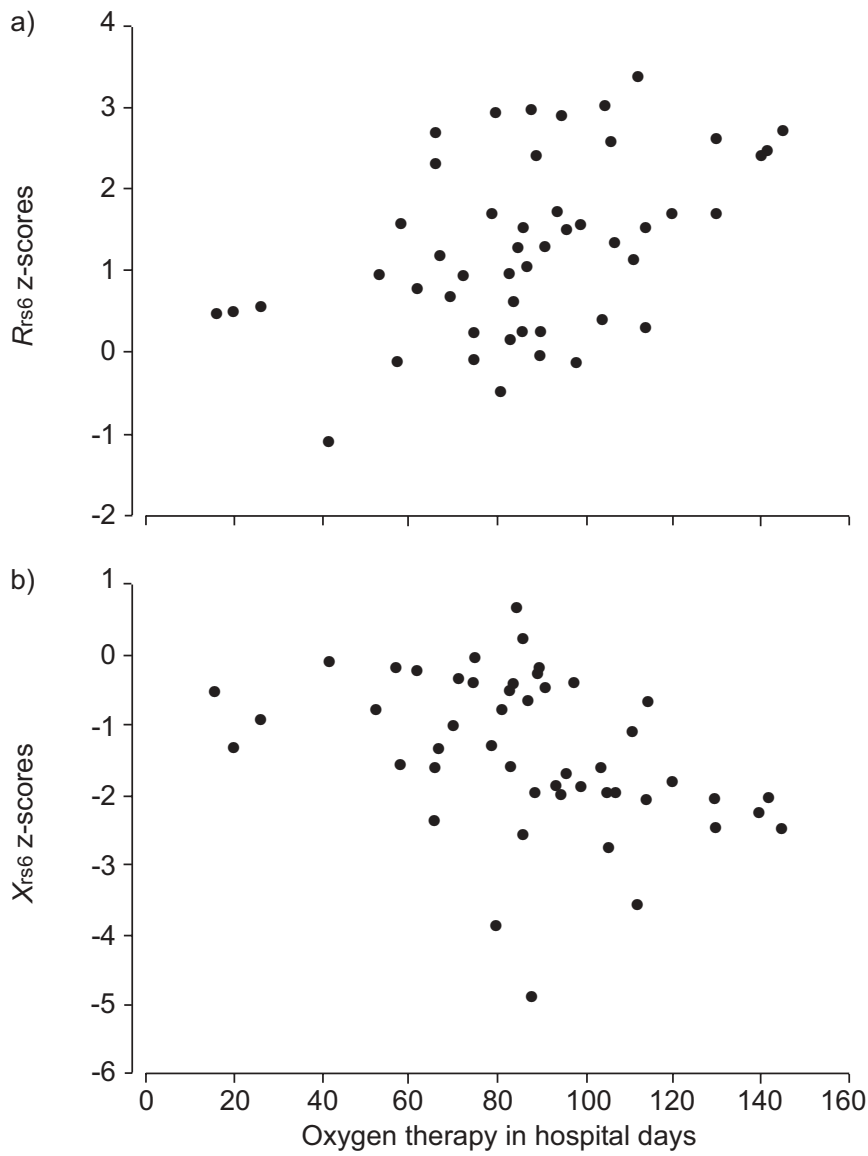

FIGURE 3. Correlation between duration of oxygen therapy in hospital in the neonatal period and a) respiratory resistance at $6 \mathrm{~Hz}$ (Rrs6) and b) respiratory reactance at $6 \mathrm{~Hz}\left(\mathrm{Xr}_{\mathrm{rs}}\right)$, assessed at age $3-7 \mathrm{yrs}$.

stages of lung development [31]. Normal growth and development is disrupted by premature birth and subsequent airway and lung injury, and this may account for respiratory dysfunction in later life [4]. In the present study, approximately one quarter of the children had abnormal lung function (i.e. outside the upper or lower limits of normal for Rrs and Xrs, respectively) and those children with abnormal lung function had received significantly more supplemental oxygen therapy $(\mathrm{p}<0.03$ and $\mathrm{p}<0.001$ for Rrs6 and Xrs6, respectively). These results would suggest abnormal lung function as defined by FOT z-scores is related to the severity of neonatal lung disease. Longitudinal studies are required to define the role of FOT in assessing the efficacy of inhaled therapies in this group of patients. There are few reports characterising the lung function of patients with nCLD beyond the first 2 yrs of life, particularly the associations between neonatal events and later lung function in individual subjects with a history of nCLD. Studies in school-aged children and adolescents with a history of nCLD have demonstrated relationships between neonatal treatments and lung function later in life [32], although these studies are primarily in those patients described as having "classic" nCLD.

The present study is the first to examine the relationships between clinical correlates of neonatal disease severity and subsequent lung function in young children with "new"
nCLD. While the current authors suggest that abnormalities in $X$ rs seen in the present study are a reflection of both the severity and pathology of "new" nCLD, this cannot be directly confirmed. In a study of infants with "new" nCLD, MAHUT et al. [33] reported a dissociation between airway function, as measured by maximal flow at function residual capacity, and computed tomographic abnormalities, suggesting a decreased airway involvement in the pathology of "new" nCLD. Studies combining new lung imaging approaches in young children [34] and lung function methods sensitive to peripheral mechanics, such as FOT, are required to address these issues in detail. In particular, techniques capable of determining alveolar surface area in vivo are likely to be informative. However, without such data, interpretations of changes in lung function will remain speculative.

\section{Study limitations}

The results of the present study have demonstrated that FOT can be used as a clinical tool to objectively measure lung function, with acceptable measurements obtained in $>75 \%$ of children aged $\geqslant 4$ yrs. The feasibility of obtaining acceptable FOT measurements in differing patient cohorts may differ and be related to age at presentation, training of children [26], disease history and/or severity of developmental delay associated with pre-term birth. The children studied were part of a follow-up service for infants with nCLD discharged from neonatal tertiary care while still receiving supplemental oxygen and, therefore, represent a group of children at the moderate-to-severe part of the nCLD spectrum [20]. It would therefore be expected that the proportion of successful FOT measurements would be higher in children with mild nCLD or those studied in a nonclinical environment.

The primary aim of the present study was to document the feasibility of using FOT to determine respiratory function in children with a history of nCLD in a clinical setting. As a result, the present authors cannot comment on the presence or severity of respiratory dysfunction in pre-term children without nCLD or with differing disease severity to those in the present study. As the children studied were presenting at respiratory outpatient clinics, information on a number of antenatal and post-natal factors known to influence respiratory health could not be comprehensively documented, including atopy and in utero and environmental tobacco exposure. Thus, while the present study demonstrated an influence of neonatal oxygen treatment on subsequent respiratory function, other factors cannot be excluded from influencing lung function at preschool age. Longitudinal studies of infants and children with nCLD are required to address these important issues and provide important information for future healthcare planning in this patient group.

In conclusion, the present study has demonstrated that the forced oscillation technique is feasible and repeatable in young children with a history of neonatal chronic lung disease. Compared with healthy preschool children, children with neonatal chronic lung disease have poorer lung function with higher respiratory resistance and lower respiratory reactance. Duration of oxygen therapy in hospital was found to be significantly associated with respiratory function during preschool age. It can be concluded that the forced oscillation technique is a simple, repeatable technique capable of 
providing clinically useful measurements of respiratory function in young children with a history of neonatal chronic lung disease. Importantly, data from the present study suggest that respiratory function as assessed by the forced oscillation technique is reflective of the severity of lung disease during the neonatal period.

\section{REFERENCES}

1 Northway WH Jr, Rosan RC, Porter DY. Pulmonary disease following respirator therapy of hyaline-membrane disease. Bronchopulmonary dysplasia. N Engl J Med 1967; 276: 357-368.

2 Bancalari E, Claure N, Sosenko IR. Bronchopulmonary dysplasia: changes in pathogenesis, epidemiology and definition. Semin Neonatol 2003; 8: 63-71.

3 Coalson JJ. Pathology of new bronchopulmonary dysplasia. Semin Neonatol 2003; 8: 73-81.

4 Eber E, Zach MS. Long term sequelae of bronchopulmonary dysplasia (chronic lung disease of infancy). Thorax 2001; 56: 317-323.

5 Baraldi E, Filippone M, Trevisanuto D, Zanardo V, Zacchello F. Pulmonary function until two years of life in infants with bronchopulmonary dysplasia. Am J Respir Crit Care Med 1997; 155: 149-155.

6 Farstad T, Brockmeier F, Bratlid D. Cardiopulmonary function in premature infants with bronchopulmonary dysplasia-a 2-year follow up. Eur J Pediatr 1995; 154: 853-858.

7 Mallory GB Jr, Chaney H, Mutich RL, Motoyama EK. Longitudinal changes in lung function during the first three years of premature infants with moderate to severe bronchopulmonary dysplasia. Pediatr Pulmonol 1991; 11: 8-14.

8 Tepper RS, Morgan WJ, Cota K, Taussig LM. Expiratory flow limitation in infants with bronchopulmonary dysplasia. J Pediatr 1986; 109: 1040-1046.

9 Baraldi E, Bonetto G, Zacchello F, Filippone M. Low exhaled nitric oxide in school-age children with bronchopulmonary dysplasia and airflow limitation. Am J Respir Crit Care Med 2005; 171: 68-72.

10 Filippone M, Sartor M, Zacchello F, Baraldi E. Flow limitation in infants with bronchopulmonary dysplasia and respiratory function at school age. Lancet 2003; 361: 753-754.

11 Malmberg LP, Mieskonen S, Pelkonen A, Kari A, Sovijärvi AR, Turpeinen M. Lung function measured by the oscillometric method in prematurely born children with chronic lung disease. Eur Respir J 2000; 16: 598-603.

12 Pelkonen AS, Hakulinen AL, Turpeinen M. Bronchial lability and responsiveness in school children born very preterm. Am J Respir Crit Care Med 1997; 156: 1178-1184.

13 Doyle LW, Cheung MM, Ford GW, Olinsky A, Davis NM, Callanan C. Birth weight $<1501 \mathrm{~g}$ and respiratory health at age 14. Arch Dis Child 2001; 84: 40-44.

14 Koumbourlis AC, Motoyama EK, Mutich RL, Mallory GB, Walczak SA, Fertal K. Longitudinal follow-up of lung function from childhood to adolescence in prematurely born patients with neonatal chronic lung disease. Pediatr Pulmonol 1996; 21: 28-34.
15 Oostveen E, MacLeod D, Lorino $\mathrm{H}$, et al. The forced oscillation technique in clinical practice: methodology, recommendations and future developments. Eur Respir J 2003; 22: 1026-1041.

16 Ducharme FM, Davis GM. Measurement of respiratory resistance in the emergency department: feasibility in young children with acute asthma. Chest 1997; 111: 1519-1525.

17 Hall GL, Sly PD, Fukushima T, et al. Respiratory function in healthy young children using forced oscillations. Thorax 2007; 62: 521-526.

18 Malmberg LP, Pelkonen A, Poussa T, Pohianpalo A, Haahtela T, Turpeinen M. Determinants of respiratory system input impedance and bronchodilator response in healthy Finnish preschool children. Clin Physiol Funct Imaging 2002; 22: 64-71.

19 Vrijlandt EJ, Boezen HM, Gerritsen J, Stremmelaar EF, Duiverman EJ. Respiratory health in prematurely born preschool children with and without bronchopulmonary dysplasia. J Pediatr 2007; 150: 256-261.

20 Jobe AH, Bancalari E. Bronchopulmonary dysplasia. Am J Respir Crit Care Med 2001; 163: 1723-1729.

21 Cole TJ, Freeman JV, Preece MA. British 1990 growth reference centiles for weight, height, body mass index and head circumference fitted by maximum penalized likelihood. Stat Med 1998; 17: 407-429.

22 Beydon N, Davis SD, Lombardi E, et al. An official American Thoracic Society/European Respiratory Society statement: pulmonary function testing in preschool children. Am J Respir Crit Care Med 2007; 175: 1304-1345.

23 Thamrin C, Gangell CL, Udomittipong K, et al. Assessment of bronchodilator responsiveness in preschool children using forced oscillations. Thorax 2007; 62: 814-819.

24 Bland JM, Altman DG. Statistical methods for assessing agreement between two methods of clinical measurement. Lancet 1986; 1: 307-310.

25 Klug B, Bisgaard H. Specific airway resistance, interrupter resistance, and respiratory impedance in healthy children aged 2-7 years. Pediatr Pulmonol 1998; 25: 322-331.

26 Gangell CL, Horak F Jr, Patterson HJ, Sly PD, Stick SM, Hall GL. Respiratory impedance in children with cystic fibrosis using forced oscillations in clinic. Eur Respir J 2007; 30: 892-897.

27 Ducharme FM, Davis GM. Respiratory resistance in the emergency department: a reproducible and responsive measure of asthma severity. Chest 1998; 113: 1566-1572.

28 Ducharme FM, Davis GM, Ducharme GR. Pediatric reference values for respiratory resistance measured by forced oscillation. Chest 1998; 113: 1322-1328.

29 Bisgaard H, Klug B. Lung function measurement in awake young children. Eur Respir J 1995; 8: 2067-2075.

30 Klug B, Bisgaard H. Measurement of lung function in awake 2-4-year-old asthmatic children during methacholine challenge and acute asthma: a comparison of the impulse oscillation technique, the interrupter technique, and transcutaneous measurement of oxygen versus wholebody plethysmography. Pediatr Pulmonol 1996; 21: 290-300.

31 Hislop AA. Airway and blood vessel interaction during lung development. J Anat 2002; 201: 325-334.

32 Kennedy JD. Lung function outcome in children of premature birth. J Paediatr Child Health 1999; 35: 516-521. 
33 Mahut B, De Blic J, Emond S, et al. Chest computed tomography findings in bronchopulmonary dysplasia and correlation with lung function. Arch Dis Child Fetal Neonatal Ed 2007; 92: F459-F464.
34 Long FR, Williams RS, Adler BH, Castile RG. Comparison of quiet breathing and controlled ventilation in the highresolution CT assessment of airway disease in infants with cystic fibrosis. Pediatr Radiol 2005; 35: 1075-1080. 